\title{
Sistema de monitoreo y control de proyectos integradores en el ámbito académico
}

\section{Monitoring and control system of integrating projects in the academic field}

PÉREZ-TORRES, Roxana †, VARGAS-VILCHIS, Emmanuel, REYES-DE LOS SANTOS, Iyeliz y VÁZQUEZ-SALAZAR, Roberto Salomón

Universidad Tecnológica del Valle de Toluca. Carretera, Del Depto del Distrito Federal km 7.5, 52044 Santa María Atarasquillo, Méx.

ID $1^{\mathrm{er}}$ Autor: Roxana, Pérez-Torres / ORC ID: 0000-0003-4191-2332, CVU CONACYT ID: 45497

ID $1^{\mathrm{er}}$ Coautor: Emmanuel, Vargas-Vilchis / ORC ID: 0000-0002-4965-978

ID $2^{\text {do }}$ Coautor: Iveñiz, Reyes-De los Santos / ORC ID: 0000-0002-8261-7906, CVU CONACYT ID: 269534

ID $3^{\text {er }}$ Coautor: Roberto Salomón, Vázquez-Salazar / ORC ID: 0000-0002-5188-784X

DOI: $10.35429 /$ JOTE.2019.8.3.19.22

Recibido 10 de Abril, 2019; Aceptado 25 de Junio, 2019

\begin{abstract}
Resumen
En este trabajo se presenta el desarrollo de un sistema informático, cuyo objetivo es, automatizar el trabajo de monitoreo de entregables de proyectos, en el ámbito escolar, en los cuales estudiantes y profesores de distintas asignaturas son involucrados principales. En el sistema cada profesor puede detallar los entregables de su asignatura, la fecha de entrega y asignar calificación. En el caso del profesor responsable, que funge como administrador, puede obtener un reporte en Excel, por grupo, de todos los entregables, de manera que pueda detectar oportunamente atrasos $y$, actuar en consecuencia. La estructura del sistema permite dar de alta cada proyecto, con la competencia a demostrar y los criterios de desempeño que están declarados en un Plan de estudios. Adicionalmente en la página inicial se muestran los entregables cuya fecha de entrega está próxima a vencer. El desarrollo del sistema se desarrolló bajo la metodología Scrum, y se utilizó Laravel como ambiente de desarrollo y actualmente está en línea.
\end{abstract}

Sistema informático, Administración de proyectos, Monitoreo

\begin{abstract}
This paper presents the development of a computer system, whose objective is to automate the work of monitoring project deliverables, in the school, in which students and professors of different subjects are mainly involved. In the system each teacher can detail the deliverables of their subject, the delivery date and assign a grade. In the case of the responsible teacher, who acts as an administrator, you can obtain a report in Excel, by group, of all the deliverables, so that you can detect timely delays and, act accordingly. The structure of the system allows to register each project, with the competence to demonstrate and the performance criteria that are declared in a Curriculum. Additionally, the initial page shows the deliverables whose delivery date is close to expiration. The development of the system was developed under the Scrum methodology, and Laravel was used as a development environment and is currently online.
\end{abstract}

Computer system, Project management, Monitoring

Citación: PÉREZ-TORRES, Roxana, VARGAS-VILCHIS, Emmanuel, REYES-DE LOS SANTOS, Iyeliz y VÁZQUEZSALAZAR, Roberto Salomón. Sistema de monitoreo y control de proyectos integradores en el ámbito académico. Revista de Educación Técnica. 2019. 3-8: 19-22. 


\section{Introducción}

El aprendizaje basado en proyectos (ABP) tuvo su inicio en el constructivismo, es una estrategia que permite integrar lo aprendido y resolver situaciones cercanas a la realidad.

El ABP representa una oportunidad para que los estudiantes logren un aprendizaje significativo, tanto en el saber, en el saber hacer $y$ en el ser, dotándolos de experiencias memorables de la aplicación de sus capacidades y habilidades tanto, técnicas como personales. Los invita a la reflexión, al manejo de información, al trabajo en equipo y sobre todo les permite crear un resultado medible, observable de lo que son capaces de hacer, ayudando a su seguridad y clarificando posibles escenarios de su vida laboral.

Si bien el ABP se puede utilizar en una sola asignatura, cuando el proyecto implica la participación de varias materias, representa un reto adicional, no solo para los estudiantes, también para los docentes.

Los docentes suelen trabajar de forma aislada, produciendo experiencias en los estudiantes en una sola línea del conocimiento, dificultando el desarrollo integral de los estudiantes. Es de suma importancia que los docentes valoren la conveniencia de trabajar conjuntamente para lograr que los estudiantes desarrollen conexiones cognitivas ante situaciones reales, generando un aprendizaje profundo promoviendo la solución de problemas, que puedan ser transferidos a nuevas situaciones.

Cuando los docentes trabajan en forma coordinada, viendo el resultado del proyecto como un todo, se puede lograr en los estudiantes el resultado esperado. Es aquí donde radica el valor del presente trabajo, ya que el sistema informático que se presenta, está diseñado para administrar de forma organizada e integrada los entregables de los proyectos, colocando como punto de partida las competencias de egreso de un plan educativo. Su organización está acorde con los planes de estudio de educación basados en competencias profesionales, en particular se describe su uso en la carrera de Ingeniería en Tecnologías de la Información y Comunicación (ITIC) en la Universidad Tecnológica del Valle de Toluca (UTVT), en el Estado de México, México.
En los programas educativos (PE) basados en competencias profesionales, en el subsistema de Universidades Tecnológicas en México, tanto en sus PE de Técnico Superior Universitario, como en su continuidad de estudios, para el nivel licenciatura, incluye dos asignaturas, de nombres Integradora I y II, cuya función es evaluar las competencias declaradas en el plan de estudios, esto está establecido en los criterios generales para la planeación, el desarrollo y la evaluación de la Coordinación General de Universidades Tecnológicas y Politécnicas. En la dirección de carrera de Tecnologías de la Información y Comunicación, en la Universidad Tecnológica del Valle de Toluca, se utilizan los siguientes pasos para la valoración de la competencia, o competencias que deben ser valoradas:

1. Analizar competencia, unidades de competencia, capacidades y criterios de desempeño establecidos en el plan de estudios. Participando todos los docentes involucrados en el cuatrimestre.

2. Se establece los criterios de desempeño que pueden ser valorados con un solo proyecto. Generalmente alrededor del $80 \%$ de los criterios.

3. Se establecen comisiones para describir las características generales de los entregables que darán cuenta de los criterios de desempeño, basado en un solo proyecto.

4. Los docentes dan seguimiento, con revisión y asesoría. El profesor(a) de integradora dan seguimiento general.

Bajo este esquema se han tenido muy buenos resultados, pero no todos los equipos de estudiantes terminan con éxito sus proyectos, en general son equipos que se atrasan en el desarrollo del proyecto, por diversas razones y que no se acercan a asesorías, por lo que al tener una herramienta de monitoreo general, se pueden identificar los equipos con dificultades, con la intención de ofrecerles acompañamiento, según la causa, ya sea técnica, metodológica, o incluso ofrecerles servicio de psicopedagogía para conflictos entre integrantes de los equipos. Otro problema que se observa es que, por la cantidad de proyectos, también los profesores se atrasan en la revisión de avances, y en ocasiones detectan tardíamente a los equipos que requieren apoyo. 
El sistema permitirá no solo monitorear a los estudiantes, también a los profesores. El sistema administra por proyecto: estudiantes, docentes, competencias, criterios de desempeño, descripción de entregables, fechas de entrega y calificaciones. El sistema fue desarrollado, basado en experiencias con proyectos integradores en la UTVT. El sistema es flexible a la cantidad de entregables que se determinen como necesarios para un criterio de desempeño. Además, se pueden colocar el $100 \%$ de los criterios de desempeño, los del proyecto, y los que por su naturaleza tiene que valorarse de forma independiente. Por ejemplo, si el proyecto base es el desarrollo de un sistema informático web con comercio electrónico, y además hay un criterio de desempeño que necesita que el estudiante construya una antena, este último no pertenece al proyecto base, pero si debe estar dado de alta en el sistema para ser valorado. El sistema está desarrollado en Laravel. Para la base de datos se utilizó el gestor MySQL.

\section{Metodología}

Se utilizó la metodología SCRUM, considerada una metodología ágil, y con un alto porcentaje de éxito, ya que es flexible a cambios y el desarrollo se lleva a cado de la mano del cliente, quién forma parte del equipo de SCRUM. Basado en esta metodología, en este proyecto, el product owner es un docente con experiencia en proyectos integradores, el scrum master fue uno de los desarrolladores y el equipo de desarrollo está compuesto por tres estudiantes de la carrera de ITIC de la UTVT. Se determinaron sprint de 3 semanas, para revisar los avances, determinar el trabajo del siguiente sprint, ordenando prioritariamente e ir aumentando la funcionalidad del sistema informático.

\section{Perfiles de usuario}

El sistema considera dos tipos de usuario: administrador y profesor. El administrador es el profesor(a) responsable del proyecto integrador, en particular, en la carrera de ITIC de la UTVT, el responsable es uno de los docentes que imparten la asignatura Integradora I o II, según sea el caso. El tipo de usuario profesor, es para los profesores(as) que están impartiendo cátedra en el cuatrimestre en curso. En la imagen 1 se muestra la interface de inicio del administrador.
La interfaz del profesor solo tiene dos apartados, uno para que describa sus entregables y otro para calificar.

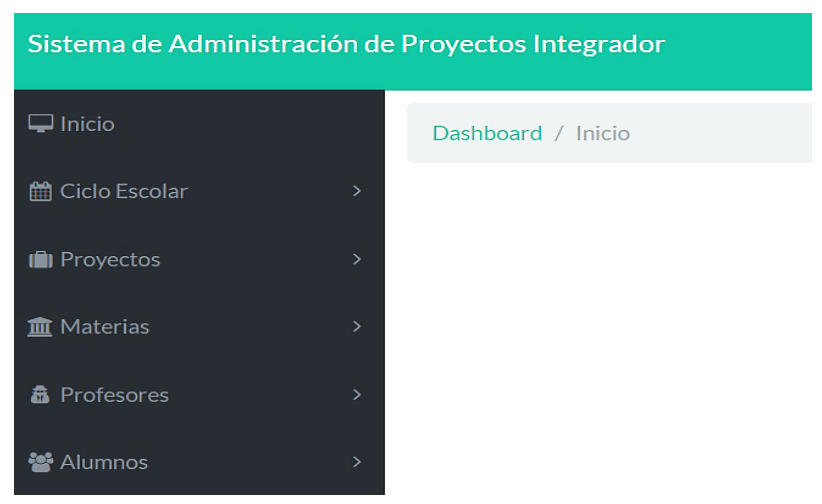

Figura 1 Interfaz del administrador

\section{Módulos}

En el apartado de ciclo escolar, el administrador da de alta el periodo escolar, por ejemplo, mayo-agosto 2019 y el cuatrimestre en el que será aplicado, tercero, quinto, noveno o décimo. Todos los demás apartados serán filtrados por estos dos datos que identifican a cada proyecto integrador general. Después se dan de alta a los grupos que participan en el proyecto. En la figura 2 se observa el alta de grupos.

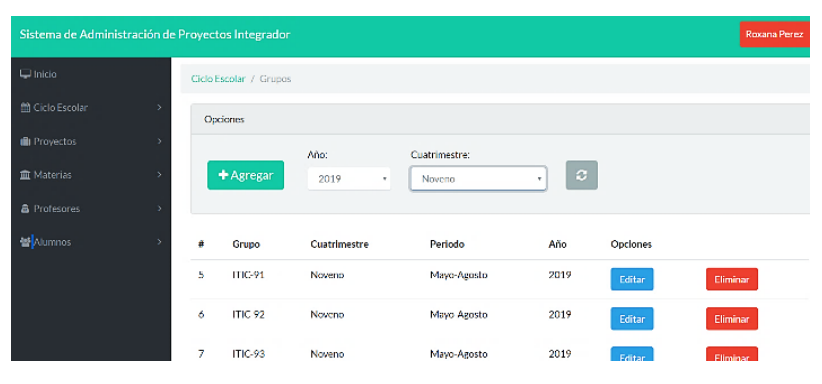

Figura 2 Alta de grupos

En el apartado de Proyectos, se establece el nombre y descripción de la competencia a evaluar, así como sus capacidades y criterios de evaluación asociadas. En la figura 3 se muestra el alta de criterios de desempeño.

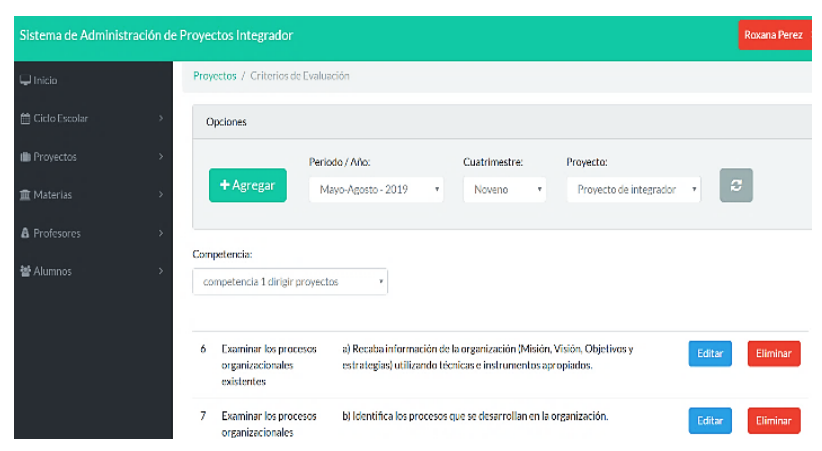

Figura 3 Alta de criterios de desempeño

PÉREZ-TORRES, Roxana, VARGAS-VILCHIS, Emmanuel, REYESDE LOS SANTOS, Iyeliz y VÁZQUEZ-SALAZAR, Roberto Salomón. Sistema de monitoreo y control de proyectos integradores en el ámbito académico. Revista de Educación Técnica. 2019 
El administrador da de alta a profesores y asignaturas, después los relaciona. Cuando se da de alta a un nuevo profesor, el sistema le envía un correo electrónico con usuario y contraseña inicial. El profesor registra entregables de su asignatura, colocando intervalo de fechas para revisión y calificación. En la figura 4 se puede observar alta de entregables de una asignatura.

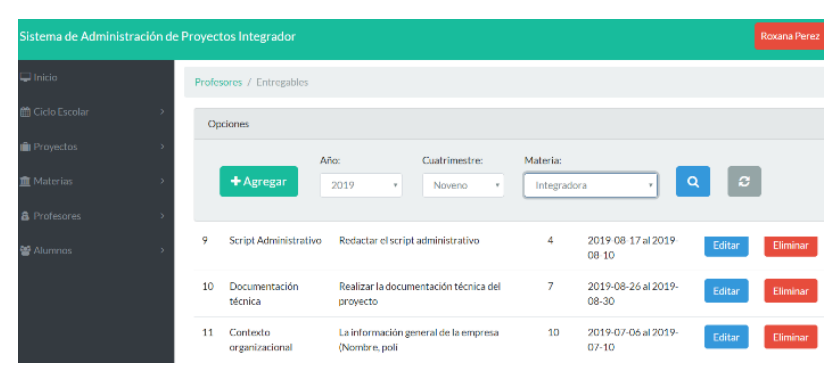

Figura 4 Alta de entregables

Cada profesor puede asignar una calificación a cada entregable por grupos, lo cual se muestra en la figura 5.

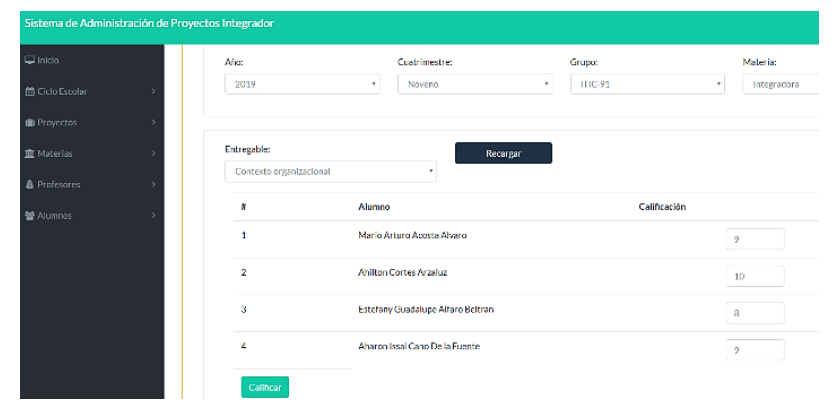

Figura 5 Calificaciones por entregable por grupo

El sistema permite descargar un reporte general de calificaciones por grupo, en Excel, además de la relación entre entregables y asignaturas. Lo cual se muestra en la figura 6.

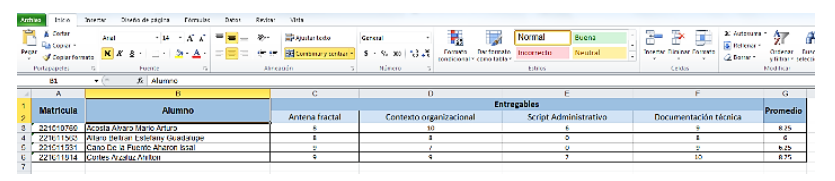

Figura 6 Reporte de calificaciones por grupo

\section{Conclusiones y trabajo futuro}

El sistema desarrollado está listo para implementarse en este cuatrimestre septiembrediciembre 2019, será analizado su funcionamiento y se realizará un plan de mejora, que incluya los hallazgos que surgen y la participación activa de los estudiantes, por ejemplo que puedan consultar sus calificaciones y que el sistema les envíe notificaciones de sus próximos entregables.
En este trabajo se hizo referencia al uso del sistema, con datos de la carrera de ITIC de la UTVT, sin embargo, el sistema es flexible y puede ser usado para cualquier proyecto, de cualquier nivel.

\section{Referencias}

Alaimo, Diego Martín. (2013). Proyectos ágiles con Scrum: flexibilidad, aprendizaje, innovación y colaboración en contextos complejos. Argentina: Kleer.

Estrada García, Aurora . (2012). El aprendizaje por proyectos y el trabajo colaborativo, como herramientas de aprendizaje, en la construcción del proceso educativo, de la Unidad de aprendizaje TIC'S. Revista Iberoamericana para la Investigación y el Desarrollo Educativo, 3, 123-138.

Morin, E. (2011). ¿Cómo vivir en tiempos de crisis? Buenos Aires: Nueva Visión.

Morin, E. (2015). Enseñar a vivir. Buenos Aires: Nueva Visión.

Pérez Torres, Roxana, Reyes de los Santos, Iyeliz, Maldonado Martínez, Abish Amparo y Rosete Fonseca, Miriam, (2018). Aprendizaje basado en proyectos integradores y propuesta de monitoreo a través de un sistema informático. Revista de Didáctica Práctica. 2018, 19.

Rodríguez, Á., Chicaiza, L., Granda, V., Reinoso, P. y Aguirre, E. (2017). ¿La indagación científica contribuye a un aprendizaje auténtico en los estudiantes? Lecturas Educación Física y Deportes. Buenos Aires, Año 21, $\mathrm{N}^{\circ}$ 224, Enero. http://www.efdeportes.com/efd224/laindagacion-cientificacontribuye-a-unaprendizaje.htm

Torres, Á. F. R., Yépez, M. M. M., \& García, N. I. C. (2019). El proyecto integrador de saberes una oportunidad para aprender a aprender. EmásF: revista digital de educación física, (57), 62-77. 